\title{
High-mass diffraction in the QCD dipole picture
}

\author{
A.Bialas, H.Navelet and R.Peschanski \\ CEA, Service de Physique Theorique, CE-Saclay \\ F-91191 Gif-sur-Yvette Cedex, France
}

July 3, 2021

\begin{abstract}
Using the QCD dipole picture of the BFKL pomeron, the crosssection of single diffractive dissociation of virtual photons at high energy and large diffractively excited masses is calculated. The calculation takes into account the full impact-parameter phase-space and thus allows to obtain an exact value of the triple BFKL Pomeron vertex. It appears large enough to compensate the perturbative 6-gluon coupling factor $\left(\frac{\alpha}{\pi}\right)^{3}$ thus suggesting a rather appreciable diffractive cross-section.
\end{abstract}

1. Among the resummation properties of the perturbative expansion of QCD, it has been shown that QCD predictions for onium-onium scattering at high energy can be obtained using the QCD dipole picture [1, 2, 3], equivalent to BFKL dynamics [4]. In these processes, an onium is considered to be a $q-\bar{q}$ state of small transverse diameter (i.e. large mass scale) $r_{0} \approx 2 / Q_{0}$. In the same framework, deep inelastic $\gamma^{*}$-onium scattering can be calculated considering a fluctuation of $\gamma^{*}$ into $q-\bar{q}$ components of transverse diameter $\bar{r} \approx 2 / Q$ and its interaction with the onium through BFKL dynamics. Interestingly enough, $q-\bar{q}$-onium scattering with two a-priori different scales $r_{0}$

\footnotetext{
* On leave from Institute of Physics, Jagellonian University, Reymonta 4, 30-059 Cracow, Poland
} 
and $\bar{r}$ leads to a pattern of scaling violations of the onium structure functions at small $x_{B j}$ similar [5] to those observed with the proton at HERA.

Indeed, it is known since some time that the data on total $\gamma^{*}-p$ crosssection [6] can be successfully described [5] by the formula derived [5, 7] in the QCD dipole picture

$$
F_{2}\left(x_{B j}, Q^{2}\right)=\frac{11 \pi}{64} N \alpha^{2} e_{f}^{2} n_{e f f} e^{\Delta_{P} Y}\left(\frac{2 a(Y)}{\pi}\right)^{\frac{1}{2}} \frac{Q r_{0}}{2} e^{-\frac{a(Y)}{2} \ln ^{2}\left(r_{0} Q / 2\right)}
$$

where

$$
Y=\ln \left(\frac{c}{x_{B j}}\right)
$$

and $c$ is a constant fixing the scale of rapidity in the process. Furthermore,

$$
a(Y)=\frac{\pi}{7 \alpha N \zeta(3) Y}, \quad 1+\Delta_{P}=1+\frac{4 \alpha N}{\pi} \ln 2
$$

is the BFKL pomeron intercept, $\alpha$ is the strong coupling constant (treated as a fixed number), $N=3$ is the number of colours and $e_{f}^{2}$ is the sum of the squares of charges of the quarks participating in the process. $n_{\text {eff }}$ is the effective number of dipoles in the proton and $r_{0}$ is their average transverse diameter. Eq.(11) contains 4 unknown parameters: $\Delta_{P}, c, n_{\text {eff }}$ and $r_{0}$. Three of them can be fixed by a fit to the data [5, 8].

2. This success of the dipole picture invites one to apply it to other diffractive processes. The high-mass diffractive dissociation of virtual photons is both theoretically [2, 9, 10] and experimentally [11] an attractive case to study. In a recent paper by two of us [9] a formula has been derived for this cross-section in the limit of large impact parameters. This allowed to study its general properties but, unfortunately, could not give a well justified normalization of the integrated cross-section which is being measured in experiment. Indeed, it was shown already for the total $\gamma^{*}$-onium cross-section that the region of impact parameter comparable to that of the two colliding onia plays an important role in the process and must be treated with a great care [12]. We shall see below that the same is true for the diffractive process we consider.

\footnotetext{
${ }^{1}$ The presently accepted values are [8]: $\Delta_{P}=.282, \quad c=1.75, \quad n_{\text {eff }} e_{f}^{2}=3.8, \quad Q_{0} \equiv$ $\frac{2}{r_{0}}=.522 \mathrm{GeV}$.
} 
In the present investigation we calculate the cross-section directly, without relying on the large impact parameter approximation. For the diffractive structure function we obtain

$$
\begin{aligned}
& \frac{d F_{T, L}^{D}}{d^{2} k}= \frac{Q^{2}}{4 \pi^{2} \alpha_{e m}} x_{P}^{-1} \frac{d \sigma\left(x_{B j}, x_{P}, Q^{2}, k\right)}{d y d^{2} k}= \\
& \frac{\alpha^{5} N^{2} e_{f}^{2}}{8 \pi^{7}} n_{e f f}^{2} e^{2 \Delta_{P} y} x_{P}^{-1}\left(\frac{2 a(y)}{2 \pi}\right)^{3} \\
& \times r_{e f f}^{2}(k) e^{-a(y) \ln ^{2}\left(k r_{e f f}(k) / 2\right)} \int \frac{d \bar{\gamma}}{2 \pi i}\left(\frac{Q}{k}\right)^{2-\bar{\gamma}} \beta^{-\Delta(\bar{\gamma})} g_{3 \mathcal{P}}(\bar{\gamma}) S_{T, L}(\bar{\gamma}),
\end{aligned}
$$

where

$$
\begin{gathered}
\Delta(\gamma)=\frac{\alpha N}{\pi}\{2 \psi(1)-\psi(1-\gamma / 2)-\psi(\gamma / 2)\} \\
S_{L}(\gamma)=\frac{2 \Gamma^{4}(1+\gamma / 2) \Gamma^{2}(2-\gamma / 2)}{\Gamma(4-\gamma) \Gamma(2+\gamma)} \frac{\Gamma(1-\gamma / 2)}{\Gamma(\gamma / 2)}, \quad \frac{S_{T}}{S_{L}}=\frac{(1+\gamma / 2)(2-\gamma / 2)}{\gamma(1-\gamma / 2)}
\end{gathered}
$$

describe the coupling to the photon and

$$
y=\ln \left(\frac{c}{x_{P}}\right), \quad \frac{x_{B j}}{x_{P}} \equiv \beta=\frac{Q^{2}}{M^{2}+Q^{2}} .
$$

$g_{3 \mathcal{P}}$ is the multidimensional integral related to the triple BFKL Pomeron interaction [13]:

$$
g_{3 \mathcal{P}}(\bar{\gamma})=\int \frac{d^{2} x}{\left|x_{+}\right|\left|x_{-}\right|} \int \frac{d^{2} s}{\left|s_{+}\right|\left|s_{-}\right|} \int \frac{d^{2} s^{\prime}}{\left|s_{+}^{\prime}\right|\left|s_{-}^{\prime}\right|}\left|s_{-} s_{-}^{\prime}\right|^{\bar{\gamma}-2}
$$

with

$$
x_{ \pm}=x \pm \frac{n}{2}, \quad s_{ \pm}=s \pm \frac{x_{+}}{2}, \quad s_{ \pm}^{\prime}=s^{\prime} \pm \frac{x_{-}}{2} .
$$

$n$ being an arbitrary unit vector.

$r_{\text {eff }}(k)$ describes the Pomeron coupling to the target,

$$
r_{e f f}(k)=\int r d^{2} r d^{2} s e^{i k \cdot s} \Phi(r, s) W(k, r, 1),
$$

where $\Phi(r, s)$ is the normalized distribution of transverse sizes $r$ and positions $s$ of the dipoles in the target. Finally,

$$
W(k, x, \gamma)=\int d^{2} s e^{-i k s}\left(\left|s+\frac{x}{2} \| s-\frac{x}{2}\right|\right)^{-\gamma}
$$


is the Fourier transform of the conformal eigenvectors, which give the exact solution of the BFKL equation in impact-parameter-space [14].

In the interesting triple-pomeron limit $\beta \rightarrow 0$ one can evaluate the path integral in (田) by the saddle point method (at $\bar{\gamma} \approx 1$ ) with the result

$$
\begin{array}{r}
\frac{d F_{T, L}^{D}}{d^{2} k}=h_{T, L} \frac{\alpha^{5} N^{2}}{2^{11} \pi^{4}} e_{f}^{2} n_{e f f}^{2} r_{e f f}^{2}(k) e^{-a(y) \ln ^{2}\left(k r_{e f f}(k) / 2\right)} g_{3 \mathcal{P}}(1) \\
\frac{Q}{k} e^{2 \Delta_{P} y} x_{P}^{-1}\left(\frac{2 a(y)}{\pi}\right)^{3}\left(\frac{2 a(Y-y)}{\pi}\right)^{\frac{1}{2}} \beta^{-\Delta_{P}} e^{-\frac{a(Y-y)}{2} \ln ^{2}(Q / k)}
\end{array}
$$

where $h_{T}=9 / 2, h_{L}=1$. The triple-Pomeron integral $g_{3 \mathcal{P}}(1)$ has been calculated either analytically by two different methods [15, 16] or by numerical Monte-Carlo integration [17]. The analytical methods yield

$$
\begin{aligned}
& g_{3 \mathcal{P}}(1) \equiv 16 \pi^{5}{ }_{4} F_{3}\left(\frac{1}{2}, \frac{1}{2}, \frac{1}{2}, \frac{1}{2} ; 1,1,1 ; 1\right) \times \\
& \times\left.\frac{\partial}{\partial \epsilon} \frac{\Gamma\left(\frac{1}{2}-\epsilon\right)}{\Gamma(1-\epsilon)}{ }_{4} F_{3}\left(\frac{1}{2}-\epsilon, \frac{1}{2}, \frac{1}{2}, \frac{1}{2} ; 1-\epsilon, 1,1 ; 1\right)\right|_{\epsilon=0} \approx 7766
\end{aligned}
$$

where ${ }_{4} F_{3}$ is the generalized hypergeometric function.

3. Let us now outline the derivation of Eqn.(1). For clarity we restrict ourselves to the case of a simple dipole target of transverse diameter $r_{0}$. The generalization to a general target (onium, proton, etc...) can be done on the same lines, as indicated at the end of this section.

Following [2], one writes

$$
\frac{d \sigma}{d y d^{2} k}=(2 \pi)^{-2} \tilde{F}_{d}\left(\bar{r}, r_{0}, Y, y, k\right)
$$

with

$$
\begin{array}{r}
\tilde{F}_{d}\left(\bar{r}, r_{0}, Y, y, k\right)=\int \frac{d x}{x} \frac{d x^{\prime}}{x^{\prime}} \frac{d \bar{x}}{\bar{x}} \frac{d \bar{x}^{\prime}}{\bar{x}^{\prime}} \tau(x, \bar{x}) \tau\left(x^{\prime}, \bar{x}^{\prime}\right) d^{2} s d^{2} s^{\prime} \\
\tilde{n}_{1}\left(r_{0}, x, y *, k\right) \tilde{n}_{1}\left(r_{0}, x^{\prime}, y *,-k\right) \tilde{n}_{2}\left(\bar{r}, \bar{x}, \bar{x}^{\prime}, Y-y *, y-y *, k\right),
\end{array}
$$

where $\tilde{n}_{1}$ and $\tilde{n}_{2}$ are single and double dipole densities in the colliding dipoles with rapidities $y *$ and $Y-y *$ respectively (note that the final result is independent of $y *) . \tau$ is the two-gluon exchange dipole-dipole amplitude. 
The equation for $\tilde{n}_{2}$ is given in [2] (Eq.52). It can be solved by the methods of Refs. [3, 15] with the result

$$
\begin{array}{r}
\tilde{n}_{2}\left(\bar{r}, \bar{x}, \bar{x}^{\prime}, Y, y, k\right)=\frac{\alpha N}{\pi^{2}} \int \frac{d \bar{\gamma}}{2 \pi i} \bar{r}^{\bar{\gamma}} e^{\Delta(\bar{\gamma})(Y-y)} \\
\int \frac{d x_{01}}{x_{01}} x_{01}^{-\bar{\gamma}} \int \frac{d^{2} x_{2} x_{01}^{2}}{x_{12}^{2} x_{02}^{2}} e^{i k x_{01} / 2} \tilde{n}_{1}\left(x_{02}, \bar{x}, y, k\right) \tilde{n}_{1}\left(x_{12}, \bar{x}^{\prime}, y, k\right)
\end{array}
$$

where the complex integral over $\bar{\gamma}$ goes from $1-i \infty$ to $1+i \infty$.

To calculate the integrals over $x, x^{\prime}, \bar{x}, \bar{x}^{\prime}, s, s^{\prime}$ we use twice the identity [19]

$$
\tilde{F}^{(1)}\left(x_{01}, x_{01}^{\prime}, Y, k\right)=\int \frac{d x}{x} \frac{d x^{\prime}}{x^{\prime}} \tau\left(x, x^{\prime}\right) n_{1}\left(x_{01}, x, Y-y, k\right) n_{1}\left(x_{01}^{\prime}, x^{\prime}, y, k\right)
$$

where $\tilde{F}^{(1)}\left(x_{01}, x_{01}^{\prime}, Y, k\right)$ is the dipole-dipole amplitude at fixed momentum transfer. We thus obtain

$$
\begin{aligned}
\tilde{F}_{d}\left(\bar{r}, r_{0}, Y, y, k\right)= & \frac{\alpha N}{\pi^{2}} \int \frac{d \bar{\gamma}}{2 \pi i} \bar{r}^{\bar{\gamma}} e^{\frac{\alpha N}{\pi} \chi(\bar{\gamma})(Y-y)} \frac{1}{2 \pi} \int \frac{d^{2} x_{01}}{x_{01}^{2}} x_{01}^{-\bar{\gamma}} e^{i k x_{01} / 2} \\
& \int \frac{d^{2} x_{2} x_{01}^{2}}{x_{12}^{2} x_{02}^{2}} \tilde{F}^{(1)}\left(r_{0}, x_{02}, y, k\right) \tilde{F}^{(1)}\left(r_{0}, x_{12}, y,-k\right)
\end{aligned}
$$

where 12 :

$$
\begin{array}{r}
\tilde{F}^{(1)}\left(r, r^{\prime}, y, k\right)=-\frac{\alpha^{2}}{\pi} r r^{\prime} \int \frac{d \gamma}{2 \pi i} e^{\Delta(\gamma) y}\left(\frac{r}{r^{\prime}}\right)^{\gamma-1}(1-\gamma)^{2} h(\gamma) \\
W(k, r, \gamma) W\left(k, r^{\prime}, 2-\gamma\right)
\end{array}
$$

with

$$
h(\gamma)=\frac{4}{(2-\gamma)^{2} \gamma^{2}} .
$$

Here it is worth to stress that the formula (19) with $W(k, x, \gamma)$ given by (I1) is exact (within the approximations inherent in the dipole approach). It is at this point that we deviate from the treatment of Ref.[9] where, following [3] the factor $\left(\left|s+\frac{x}{2} \| s-\frac{x}{2}\right|\right)^{-\gamma}$ in (11) was replaced by its asymptotic form $|s|^{-2 \gamma}$. This simplification allowed a first estimate of the solution but, while removing the singularities at $s= \pm \frac{x}{2}$, it changed the absolute value of the integral. 
Substituting (19) into (18) we obtain

$$
\begin{array}{r}
\tilde{F}_{d}\left(\bar{r}, r_{0}, Y, y, k\right)=\frac{\alpha^{5} N}{\pi^{4}}\left(r_{0}\right)^{4} \int \frac{d \bar{\gamma}}{2 \pi i} \bar{r} \bar{\gamma} e^{\Delta(\bar{\gamma})(Y-y)} \\
\int \frac{d \gamma}{2 \pi i} e^{\Delta(\gamma) y} W\left(k, r_{0}, 2-\gamma\right) r_{0}^{-\gamma}(1-\gamma)^{2} h(\gamma) \\
\int \frac{d \gamma^{\prime}}{2 \pi i} e^{\Delta\left(\gamma^{\prime}\right) y} W\left(-k, r_{0}, 2-\gamma^{\prime}\right) r_{0}^{-\gamma^{\prime}}\left(1-\gamma^{\prime}\right)^{2} h\left(\gamma^{\prime}\right) Z\left(k, \bar{\gamma}, \gamma, \gamma^{\prime}\right)
\end{array}
$$

where

$$
\begin{array}{r}
Z\left(k, \bar{\gamma}, \gamma, \gamma^{\prime}\right)=\frac{1}{2 \pi} \int \frac{d^{2} x_{01}}{x_{01}^{2}} x_{01}^{-\bar{\gamma}} e^{i k x_{01} / 2} \int d^{2} x_{2} \frac{x_{01}^{2}}{x_{12}^{2} x_{02}^{2}} \\
x_{02}^{\gamma} W\left(k, x_{02}, \gamma\right) x_{12}^{\gamma^{\prime}} W\left(-k, x_{12}, \gamma^{\prime}\right) .
\end{array}
$$

Using now (11) one can perform the integration over $d^{2} x_{01}$ and sort out the dependence of $Z$ on $k$. One obtains

$$
Z\left(k, \bar{\gamma}, \gamma, \gamma^{\prime}\right)=2^{3-\bar{\gamma}-\gamma-\gamma^{\prime}} k^{\bar{\gamma}+\gamma+\gamma^{\prime}-4} \frac{\Gamma\left(2-\frac{\bar{\gamma}+\gamma+\gamma^{\prime}}{2}\right)}{\Gamma\left(-1+\frac{\bar{\gamma}+\gamma+\gamma^{\prime}}{2}\right)} g_{3 \mathcal{P}}\left(\bar{\gamma}, \gamma, \gamma^{\prime}\right)
$$

where (c.f. definition (9))

$$
\begin{gathered}
g_{3 \mathcal{P}}\left(\bar{\gamma}, \gamma, \gamma^{\prime}\right)=\int \frac{d^{2} x}{\left|x_{+}\right|^{2-\gamma}\left|x_{-}\right|^{2-\gamma^{\prime}}} \\
\int \frac{d^{2} s}{\left(\left|s_{+}\right|\left|s_{-}\right|\right)^{\gamma}} \int \frac{d^{2} s^{\prime}}{\left(\left|s_{+}^{\prime}\right|\left|s_{-}^{\prime}\right|\right)^{\gamma^{\prime}}}\left|s_{-}-s_{-}^{\prime}\right|^{\bar{\gamma}+\gamma+\gamma^{\prime}-4}
\end{gathered}
$$

It is not difficult to modify this result to account for targets composed of dipoles. One simply has to integrate over the distribution of the dipoles in the target. Consequently Eqn. (21) becomes:

$$
\begin{array}{r}
\tilde{F}_{d}(\bar{r}, Y, y, k)=\frac{8 \alpha^{5} N}{\pi^{4}} k^{-4} \int \frac{d \bar{\gamma}}{2 \pi i}\left(\frac{k \bar{r}}{2}\right)^{\bar{\gamma}} e^{\Delta(\bar{\gamma})(Y-y)} \\
\int \frac{d \gamma}{2 \pi i} e^{\Delta(\gamma) y}\left(\frac{k r_{e f f}(k, \gamma)}{2}\right)^{\gamma}(1-\gamma)^{2} h(\gamma) \\
\int \frac{d \gamma^{\prime}}{2 \pi i} e^{\Delta\left(\gamma^{\prime}\right) y}\left(\frac{k r_{e f f}\left(k, \gamma^{\prime}\right)}{2}\right)^{\gamma^{\prime}}\left(1-\gamma^{\prime}\right)^{2} h\left(\gamma^{\prime}\right) \frac{\Gamma\left(2-\frac{\bar{\gamma}+\gamma+\gamma^{\prime}}{2}\right)}{\Gamma\left(-1+\frac{\bar{\gamma}+\gamma+\gamma^{\prime}}{2}\right)} g_{3 \mathcal{P}}\left(\bar{\gamma}, \gamma, \gamma^{\prime}\right)(25
\end{array}
$$


where

$$
r_{e f f}^{\gamma}(k, \gamma)=\int r^{2-\gamma} d^{2} r d^{2} s e^{i k \cdot s} \Phi(r, s) W(k, r, 2-\gamma) .
$$

Since the formula (25) is anyway valid only in the limit $x_{p} \rightarrow 0$, the next natural step is to evaluate the integrals over $\gamma$ and $\gamma^{\prime}$ by the saddle point method. When the result is sandwiched between the photon wave functions [20] and integrals over $\bar{r}$ and quark light-cone fractions performed, one obtains (4).

4. In conclusion, using the technique of the QCD dipole picture, we have evaluated the BFKL Pomeron contribution to high-mass diffraction dissociation of the virtual photon. The result is a compact formula which we feel - may be a good starting point for a phenomenological discussion of the photon diffractive dissociation on a proton target in a QCD framework.

Several comments are in order.

(i) Eqn. (四) was obtained by the evaluation of the inverse Mellin transforms in (25) by the saddle-point method. This is expected to be a reasonable approximation as long as $x_{P}$ is small enough. One often finds convenient, however, to have a formula for $d F_{T, L}^{D} / d^{2} k$ which exhibits explicitely the path integrals in $\gamma$ and $\gamma^{\prime}$. The result reads

$$
\begin{array}{r}
\frac{d F_{T, L}^{D}}{d^{2} k}=\frac{\alpha^{5} N^{2} e_{f}^{2} n_{e f f}^{2}}{2 \pi^{9}} k^{-2} x_{P}^{-1} \int \frac{d \bar{\gamma}}{2 \pi i}\left(\frac{Q}{k}\right)^{2-\bar{\gamma}} e^{\Delta(\bar{\gamma})(Y-y)} S_{T, L}(\bar{\gamma}) \\
\int \frac{d \gamma}{2 \pi i} e^{\Delta(\gamma) y\left(\frac{k r_{e f f}(k, \gamma)}{2}\right)^{\gamma}(1-\gamma)^{2} h(\gamma)} \\
\int \frac{d \gamma^{\prime}}{2 \pi i} e^{\Delta\left(\gamma^{\prime}\right) y}\left(\frac{k r_{e f f}\left(k, \gamma^{\prime}\right)}{2}\right)^{\gamma^{\prime}}\left(1-\gamma^{\prime}\right)^{2} h\left(\gamma^{\prime}\right) \\
\frac{\Gamma\left(2-\frac{\bar{\gamma}+\gamma+\gamma^{\prime}}{2}\right)}{\Gamma\left(-1+\frac{\bar{\gamma}+\gamma+\gamma^{\prime}}{2}\right)} g_{3 \mathcal{P}}\left(\bar{\gamma}, \gamma, \gamma^{\prime}\right)
\end{array}
$$

(ii) It is illuminating to compare these results with the one obtained by integration of the asymptotic formula in impact parameter [9], viz.

$$
\begin{gathered}
F_{\text {Tasymptotic }}^{\text {Ddipole }}=\frac{9 \pi G \alpha^{5} N^{2}}{8} e_{f}^{2} n_{\text {eff }}^{2} \\
\frac{r_{0} Q}{2} e^{2 \Delta_{P} y} x_{P}^{-1}\left(\frac{2 a(y)}{\pi}\right)^{3}\left(\frac{2 a(Y-y)}{\pi}\right)^{\frac{1}{2}} \beta^{-\Delta_{P}} e^{-\frac{a(Y-y)}{2} \ln ^{2}\left(r_{0} Q / 2\right)}
\end{gathered}
$$


$(G \approx .915$ is Catalan's constant). Equation (28) has been obtained for diffraction on a single dipole. To compare, one has to take $r_{\text {eff }}^{\gamma}=r_{0}^{2-\gamma} W\left(k, r_{0}, 2-\gamma\right)$ in formula (27), integrate over $d^{2} k$ and evaluate the path integral by the saddle-point method. The integration over $d^{2} k$ is done with help of formula

$$
\int d^{2} k k^{\bar{\gamma}-2} W\left(k, r_{0}, 1\right) W\left(-k, r_{0}, 1\right)=\pi\left(r_{0} / 2\right)^{-\bar{\gamma}} \frac{\Gamma(\bar{\gamma} / 2)}{\Gamma(1-\bar{\gamma} / 2)} U_{2}(\bar{\gamma})
$$

where

$$
U_{2}(\bar{\gamma})=\int \frac{d^{2} t}{\left|t_{+}\right|\left|t_{-}\right|} \int \frac{d^{2} t^{\prime}}{\left|t_{+}^{\prime}\right|\left|t_{-}^{\prime}\right|}\left|t-t^{\prime}\right|^{-\bar{\gamma}}
$$

with the two-dimensional vectors $t_{ \pm}$and $t_{ \pm}^{\prime}$ given by

$$
t_{ \pm}=t \pm \frac{n}{2}, \quad t_{ \pm}^{\prime}=t^{\prime} \pm \frac{n}{2}
$$

The integral $U_{2}(1)$ yields [15:

$$
U_{2}(1)=2 \pi^{4}{ }_{3} F_{2}^{2}\left(\frac{1}{2}, \frac{1}{2}, \frac{1}{2} ; 1,1 ; 1\right)=\frac{\Gamma^{8}(1 / 4)}{8 \pi^{2}} \approx 378,
$$

where ${ }_{3} F_{2}$ is the generalized hypergeometric function.

The resulting formula shows two striking features:

(a) The dependence on $Q^{2}, \beta$ and $x_{P}$ is exactly the same as in formula (28). Thus the asymptotic formula correctly reproduces the dependence of the structure function on the kinematic variables. It is most likely yet another consequence of the global conformal invariance of the theory [14].

(b) The normalization factors are very different, however, the exact approach giving a much larger diffractive cross-section than the asymptotic formula: the enhancement factor given by the ratio

$$
\frac{F^{\text {Ddipole }}}{F_{\text {asymptotic }}^{\text {Ddipole }}}=\frac{U_{2}(1) g_{3 \mathcal{P}}(1)}{128 \pi^{4} G} \approx 257
$$

is indeed formidable. This is related to the fact that the asymptotic formula ignores the strong enhancement in the singular region where the impact parameter is close to the tranverse sizes of the interacting dipoles. For other targets (e.g. a proton) this enhancement factor is modified, however, depending on the shape of the distribution $\Phi(r, s)$. 
(iii) In order to compare our result with the ordinary triple Regge formalism [22], we evaluate the effective triple Pomeron coupling emerging from Eqn.(田). For this sake, let us consider the ratio of the Mellin transforms of the diffractive and total $\gamma^{*}$-proton cross-sections, defined by $\sigma \equiv \int \frac{d \bar{\gamma}}{2 \pi i}\left(\frac{Q r_{0}}{2}\right)^{2-\bar{\gamma}} \tilde{\sigma}$. Using (田) and Ref. 21], formula (34), one obtains at the saddle-point $\bar{\gamma}=1$,

$$
\left.\frac{1}{\tilde{\sigma}(\beta)} \frac{d \tilde{\sigma}}{d y d^{2} k}\right|_{\bar{\gamma}=1}=\frac{\alpha^{3} N}{64 \pi^{5}} n_{e f f} e^{2 \Delta_{P} y}\left(\frac{2 a(y)}{\pi}\right)^{3} g_{3 \mathcal{P}}(1) \frac{2 r_{e f f}^{2}(k)}{k r_{0}} e^{-a(y) \ln ^{2}\left(\frac{k r_{e f f}(k)}{2}\right)}
$$

Eqn. (34) reveals some similarity with that obtained from Regge theory in the triple Pomeron region, which for diffractive excitation on the $\operatorname{target} B$ gives

$$
\frac{1}{\sigma} \frac{d \sigma}{d y d^{2} k}=\frac{g_{B}^{2}(k)}{16 \pi^{2}} e^{2\left(\alpha_{P}(k)-1\right) y} G_{3 \mathcal{P}}\left(k^{2}\right),
$$

where $g_{B}(k)$ is the coupling of the Pomeron to the proton and $G_{3 \mathcal{P}}\left(k^{2}\right)$ is the triple Pomeron coupling, in the case when the Pomeron is a simple Regge pole [22. One observes, however, that Eqn.(34) does not correspond to the exchange of Regge poles but contains additional logarithmic corrections. This is not surprising because the BFKL Pomeron corresponds to a Regge cut in the complex angular momentum [4].

Putting aside the differences between (34) and (35) due to the different dynamical content, we are led to estimate an effective triple Pomeron coupling as:

$$
G_{3 \mathcal{P}}^{e f f}(k) \approx \frac{1}{k} g_{3 \mathcal{P}}(1)\left(\frac{\alpha}{\pi}\right)^{3} \frac{N}{4}\left(\frac{2 a(y)}{\pi}\right)^{3} .
$$

One sees that the factor $\frac{1}{k}$, which was already observed in Ref. [2], sets the scale of $G_{3 \mathcal{P}}^{e f f}(k)$ as expected in a conformal invariant theory. On the other hand, the presence of the factor $\left(\frac{2 a(y)}{\pi}\right)^{3}$ reflects the logarithmic corrections to the Pomeron singularity and lowers the effective Pomeron intercept [9]. The factor $\left(\frac{\alpha}{\pi}\right)^{3}$ is naturally expected for the perturbative 6-gluon coupling. The value of $g_{3 \mathcal{P}}(1)$ given in (13), however, largely compensates the smallness of the perturbative factor. One thus expects a fairly large diffractive crosssection in this framework.

(iv) It should be stressed that our analysis is valid only for $k$ different from 0 . The point $k=0$ (crucial e.g. for the calculation of nuclear shadowing 
[23]) is special and thus requires a separate discussion. Note that -until nowonly the forward BFKL amplitudes were subject to a stringent experimental testing [5, 8]. Thus our calculation enlarges the possibilities of investigating the relevance of BFKL dynamics in the as yet poorly explored regime of $k$ different from 0 .

Acknowledgements. We would like to thank Ch.Royon for discussion and communicating his unpublished results. The discussions with A.Kaidalov are highly appreciated. AB thanks J.Zinn-Justin for the kind hospitality at Service de Physique Théorique de Saclay. This work was supported in part by the KBN Grant No 2 P03B083 08 and by PECO grant from the EEC Programme "Human Capital Mobility", Network "Physics at High Energy Colliders", Contract No ERBICIPDCT 940613. 


\section{References}

[1] A.H. Mueller, Nucl.Phys. B415 (1994) 373.

[2] A.H.Mueller and B.Patel, Nucl.Phys. B425 (1994) 471.

[3] A.H. Mueller, Nucl.Phys. B437 (1995) 107.

[4] L.N.Lipatov, Sov. J. Nucl. Phys. 23 (1976) 642; V.S.Fadin, E.A.Kuraev and L.N.Lipatov, Phys. lett. B60 (1975) 50; E.A.Kuraev, L.N.Lipatov and V.S.Fadin, Sov.Phys.JETP 44 (1976) 45, 45 (1977) 199; I.I.Balitsky and L.N.Lipatov, Sov.J.Nucl.Phys. 28 (1978) 822.

[5] H.Navelet, R.Peschanski and Ch.Royon, Phys.Letters B366 (1995) 329; H.Navelet, R.Peschanski, Ch.Royon and S.Wallon, Phys.Letters B385 (1996) 357.

[6] H1 Coll. T.Ahmed et al., Phys.Letters B348 (1995) 681; ZEUS Coll. M.Derrick et al., Z.Phys. C68 (1995) 569.

[7] A.Bialas, Acta Phys.Pol B27 (1996) 1263

[8] Ch.Royon, private communication.

[9] A.Bialas and R.Peschanski, Phys. Letters B378 (1966) 302.

[10] Some of the relevant references are: G.Ingelman, P.Schlein, Phys.Lett. B152 (1985) 256 N.N.Nikolaev and B.G.Zakharov, Zeit. für. Phys. C53 (1992) 331. J.D.Bjorken, preprint SLAC-PUB-7096 (1996). A.Edin, G.Ingelman, J.Rathsman, Phys.Lett. B366 (1996) 371, Z.Phys. C75 (1997) 57, W.Buchmüller, A.Hebecker, Phys.Lett. B355 (1995) 573, Nucl.Phys. B476 (1996) 203. A.Capella et al., hep-ph/9707466 and references therein.

[11] H1 Coll., T. Ahmed et al. Phys. Lett. B348 (1995) 681. ZEUS Coll., M.Derrick et al. Z. Phys. C68 (1995) 569. H1 Coll. C. Adloff et al., Preprint DESY 97-158 (1997).

[12] G.P.Salam, Nucl. Phys. B461 (1996) 512, and PHD thesis; H.Navelet and S.Wallon, hep-ph/9705296, to be published in Nucl. Phys. B. 
[13] In the BFKL framework: J.Bartels, Zeit. fur. Phys. C60 (1993) 471; Phys. Lett. B298 (1993) 204; J.Bartels, M.Wüsthoff Zeit. fur. Phys. C66 (1995) 157; J.Bartels, L.N.Lipatov, M.Wüsthoff Nucl. Phys. B464 (1996) 298. In the QCD dipole framework: R.Peschanski, Phys. Lett. B. 409 (1997) 491.

[14] L.N.Lipatov, Zh. Eksp. Teor. Fiz. 90 (1986) 1536 ( Sov. Phys. JETP 63 (1986) 904).

[15] A.Bialas, H.Navelet and R.Peschanski, to be published.

[16] G.P.Korchemsky preprint Orsay LPTHE 97-62, hep-ph/9711277[revised version].

[17] I.I.Balitsky and V.M.Braun, unpublished.

[18] A.Bialas, R.Peschanski, Phys. Lett. B355 (1995) 301.

[19] H.Navelet and R.Peschanski, hep-ph/9703238, to be published in Nucl. Phys. B.

[20] J.D.Bjorken, J.Kogut and D. Soper, Phys. Rev. D 3 (1971) 1382.

[21] A.Bialas, Acta Phys. Pol. B 28 (1997) 1239.

[22] See, for instance: A.Kaidalov, Phys. Rep. 50 (1979) 157.

[23] A.Bialas, W.Czyz and W. Florkowski, Phys. Rev. D55 (1997) 6830; A.Bialas and W.Czyz, submitted to Acta Physica Polonica B. 